Abstract

\title{
Semi-Covalent Imprinting for Selective Protein Sensing at a Femtomolar Concentration Level ${ }^{\dagger}$
}

\author{
Marcin Dabrowski ${ }^{1}$, Maciej Cieplak ${ }^{1, *}$, Piyush Sindhu Sharma ${ }^{1}$, Pawel Borowicz ${ }^{1}$, \\ Krzysztof Noworyta ${ }^{1}$, Alexander Kuhn ${ }^{2,3, *}$, Francis D'Souza ${ }^{4, *}$ and Wlodzimierz Kutner ${ }^{1,5, *}$ \\ 1 Institute of Physical Chemistry, Polish Academy of Sciences (IPC PAS), Kasprzaka 44/52, 01-224 Warsaw, \\ Poland; mdabrowski@ichf.edu.pl (M.D.); psharma@ichf.edu.pl (P.S.S.); pborowicz@ichf.edu.pl (P.B.); \\ knoworyta@ichf.edu.pl (K.N.) \\ 2 Institut des Sciences Moléculaires, University of Bordeaux, Bordeaux INP, ENSCBP, \\ 16 Avenue Pey Berland, 33607 Pessac, France \\ 3 Le Centre National de la Recherche Scientifique, Institute of Molecular Sciences, UMR 5255, \\ 351 Cours de la Liberation, 33400 Talence, France \\ 4 Department of Chemistry, University of North Texas, 1155 Union Circle, \#305070, Denton, \\ TX 76203-5017, USA \\ 5 Faculty of Mathematics and Natural Sciences, School of Sciences, Cardinal Stefan Wyszynski University in \\ Warsaw, Woycickiego 1/3, 01-815 Warsaw, Poland \\ * Correspondence: mcieplak@ichf.edu.pl (M.C.); kuhn@enscbp.fr (A.K.); francis.dsouza@unt.edu (F.D.); \\ wkutner@ichf.edu.pl (W.K.) \\ + Presented at the 5th International Symposium on Sensor Science (I3S 2017), Barcelona, Spain, \\ 27-29 September 2017.
}

Published: 4 December 2017

Protein imprinting is challenging mainly because of their large molecular size. It is very difficult to estimate which and how many groups on the protein template molecule surface are accessible for binding. To overcome this drawback, we introduced semi-covalent protein imprinting [1]. We prepared a conducting molecularly imprinted polymer (MIP) based on bis(2,2'-bithien-5-yl)methane for human serum albumin (HSA) determination. A very high imprinting factor (IF > 20) and selectivity of the devised chemosensor proved that the MIP featured molecular cavities of welldefined structure and high affinity to HSA. This success encouraged us to improve this approach even further. For that, we prepared a new artificial receptor material in the form of a thin macroporous MIP film with an unprecedented hierarchical nanostructure controlled at three different size scale levels [2]. The introduction of this nanostructure resulted in the extraordinary properties of this recognizing material. That is, its very high selectivity of MIP based extended-gate field-effect transistor (EG-FET) chemosensor was accompanied by high sensitivity and detectability at an impressive femtomolar concentration level. These analytical parameters were among the best reported in literature not only for MIP chemosensors but in the field of bio- and chemosensors in general.

\section{References}

1. Cieplak, M.; Szwabinska, K.; Sosnowska, M.; KC, C.B.; Borowicz, P.; Noworyta, K.; D’Souza, F.; Kutner, W. Selective electrochemical sensing of human serum albumin by semi-covalent molecular imprinting. Biosens. Bioelectron. 2015, 74, 960-966.

2. Dabrowski, M.; Cieplak, M.; Sharma, P.S.; Borowicz, P.; Noworyta, K.; Lisowski, W.; D’Souza, F.; Kuhn, A.; Kutner, W. Hierarchical templating in deposition of semi-covalently imprinted inverse opal polythiophene film for femtomolar determination of human serum albumin. Biosens. Bioelectron. 2017, 94, 155-161. 